\title{
Treatment Options in ErbB2 (HER2)-Overexpressing Breast Cancer
}

\author{
Christian Jackisch \\ Department of Obstetrics and Gynecology and Breast Cancer Center, Klinikum Offenbach GmbH, Germany
}

In recent years, the traditional view of breast cancer as a single-tumor entity has been challenged. Today, breast cancer is considered a heterogeneous group of different carcinomas, one of them the ErbB2-overexpressing breast tumor. Overexpression of ErbB2 (HER2), a receptor belonging to the ErbB (HER) family of tyrosine kinases, occurs in approximately 20 $25 \%$ of all breast tumors. ErbB2 overexpression is associated with an adverse prognosis characterized by short intervals to recurrence and a short overall survival. The first agent to target ErbB2 was the humanized monoclonal antibody trastuzumab. In combination with chemotherapy this targeted agent led to a dramatic improvement of treatment results in patients with metastatic ErbB2-overexpressing breast cancer. On the basis of these data, trastuzumab was evaluated in early breast cancer concomitantly with or shortly after completion of chemotherapy in the adjuvant treatment setting. In several trials, this combined adjuvant therapy resulted in a reduced risk of recurrence by about $50 \%$ and a reduced risk of death by about one third.

Lapatinib, a dual receptor tyrosine kinase inhibitor, is an alternative approach to target ErbB2-overexpressing breast cancer. This orally administered small molecule blocks ErbB2and ErbB1-mediated downstream-signaling pathways. As this novel drug has shown activity in breast cancer xenograft models expressing the truncated ErbB2 fragment p95 and in tumors with PTEN (phosphatase and tensin homologue deleted on chromosome 10) deficiency, both resistant to trastuzumab, it might represent another step forward to optimizing treatment in ErbB2-overexpressing breast cancer. In preclinical studies, the combination of trastuzumab and lapatinib demonstrated synergistic effects.
Treatment with lapatinib combined with capecitabine led to a significant increase in response rates resulting in a significant prolongation of time to progression compared to capecitabine alone in heavily pretreated patients with metastatic ErbB2overexpressing breast cancer. All patients had received anthracyclines, taxanes, and trastuzumab. Lapatinib plus paclitaxel as first-line therapy resulted in a significant improvement of time to progression versus paclitaxel monotherapy in women with ErbB2-overexpressing metastatic breast cancer. Lapatinib also has shown promising activity in the neoadjuvant therapy of patients with inflammatory breast cancer. Patients with ErbB2-overexpressing breast cancer have a high risk of central nervous system (CNS) disease of about $30 \%$. Since lapatinib has demonstrated some activity in patients with brain metastases, it is currently investigated in several trials to evaluate its potential in reduction or prevention of CNS metastases.

Based on the promising results of lapatinib in the metastatic setting, the drug is now further evaluated in early-stage breast cancer. In the TEACH trial, patients with a diagnosis of ErbB2-overexpressing breast cancer who have received adjuvant chemotherapy, but have not been treated with adjuvant trastuzumab, are randomized to lapatinib or placebo. The ALTTO trial has a four-arm design and compares monotherapy with trastuzumab or lapatinib, respectively, with the combination as well as the sequential administration of both drugs. Neo-ALTTO is a three-arm phase III trial in the neoadjuvant setting. Patients are treated with paclitaxel and are randomly assigned to additional trastuzumab or lapatinib or to the combination of both ErbB2-targeting therapies as (neo-)adjuvant treatment. The results of these trials are eagerly awaited.

\begin{tabular}{ll}
\hline KARGER & @ 2008 S. Karger GmbH, Freiburg \\
Fax +49 761 452 07 14 & Accessible online at: \\
$\begin{array}{l}\text { E-mail Information@Karger.de } \\
\text { www.karger.com }\end{array}$ & www.karger.com/brc
\end{tabular}

